\title{
Control and alignment of segmented-mirror telescopes: matrices, modes, and error propagation
}

\author{
Gary Chanan, Douglas G. MacMartin, Jerry Nelson, and Terry Mast
}

\begin{abstract}
Starting from the successful Keck telescope design, we construct and analyze the control matrix for the active control system of the primary mirror of a generalized segmented-mirror telescope, with up to 1000 segments and including an alternative sensor geometry to the one used at Keck. In particular we examine the noise propagation of the matrix and its consequences for both seeing-limited and diffractionlimited observations. The associated problem of optical alignment of such a primary mirror is also analyzed in terms of the distinct but related matrices that govern this latter problem. (C) 2004 Optical Society of America
\end{abstract}

OCIS codes: $\quad 010.7350,110.6770,120.5050,220.1140$.

\section{Introduction}

The next generation of ground-based optical and infrared telescopes is currently in the planning stages, with a variety of projects in the $20-100-m$ class currently under consideration. ${ }^{1-7}$ The majority of these telescopes involve a highly segmented primary mirror, with several hundred up to even a few thousand segments. The optimal number of segments used to fill a particular aperture size is the result of a key design trade-off: Larger segments are in general more difficult to fabricate whereas smaller segments are more difficult to align and control, principally as a result of their larger numbers.

In this paper we discuss alignment and control of highly segmented telescopes in terms of the control matrices that define these tasks. A modal analysis provides physical insight into the control and alignment problems, and we pay particular attention to the question of error propagation. When supplemented with estimates of sensor noise and measurement uncertainty, this analysis can be used to estimate the relevant terms in the optical error bud-

G. Chanan (gchanan@galaxy.ps.uci.edu) is with the Department of Physics and Astronomy, University of California, Irvine, Irvine, California 92697-4575. D. G. MacMartin is with Control and Dynamical Systems, California Institute of Technology, Pasadena, California 91125. J. Nelson and T. Mast are with University of California Observatories/Lick Observatory, University of California, Santa Cruz, Santa Cruz, California 95064.

Received 12 August 2003; revised manuscript received 17 November 2003; accepted 20 November 2003.

0003-6935/04/061223-10\$15.00/0

(C) 2004 Optical Society of America get for diffraction-limited or for seeing-limited observations and to help resolve the question of the optimal number of segments for a given aperture.

The analysis presented here is a generalization of that used for the successful control and alignment ${ }^{8,9}$ of the 36-segment primary mirrors of the two telescopes of the W. M. Keck Observatory. ${ }^{10}$ The Keck experience has led to a number of practical considerations that inform the analysis presented here.

In this paper we take the proposed 30-m California Extremely Large Telescope (CELT) as the prototype of the next generation of highly segmented telescopes. (Its current design calls for a total of 1080 segments.) An introduction to the CELT segment control matrices, position actuators, edge sensors, and error propagation can be found elsewhere. ${ }^{11-13}$ We also consider intermediate designs between Keck and CELT.

This paper is organized as follows. In Section 2 we describe the construction of and error propagation by the matrices responsible for the active control of segmented-mirror telescopes. Implications for both diffraction-limited and seeing-limited observations are discussed. In Section 3 we describe the construction of and error propagation by the matrices used in the phasing of these mirrors. Our conclusions are summarized in Section 4.

\section{Active Control}

\section{A. General Considerations}

At Keck and at other segmented-mirror telescopes, ${ }^{14,15}$ the primary mirror segments are actively positioned in their 3 out-of-plane degrees of freedom 

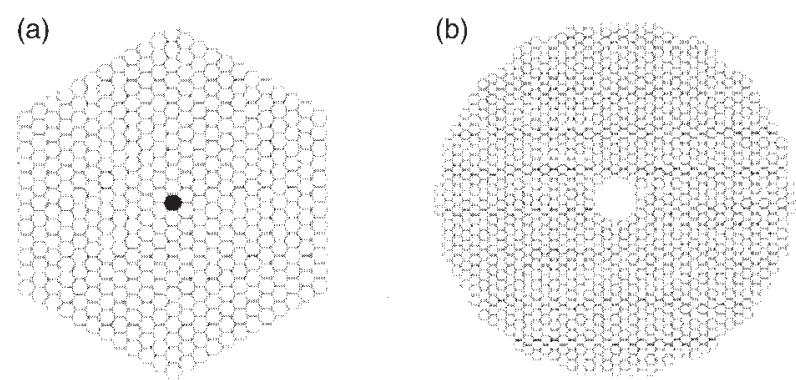

Fig. 1. Segment geometry of (a) an 11-ring telescope and (b) CELT. The 36-segment Keck geometry corresponds to the central three rings of (a).

by three mechanical actuators. (Because the optical tolerances on the in-plane degrees of freedom are considerably less restrictive, these 3 degrees of freedom are positioned passively.) The relative displacements of adjacent mirror segments are sensed by precision capacitive edge sensors, of which there are two per intersegment edge. The segments are actively controlled by means of a two-step process: (1) Initially, the desired readings of the edge sensors are determined by external optical means ${ }^{16} ;(2)$ subsequently, the mirror is stabilized against perturbations due to gravity and thermal effects by the moving the actuators so as to maintain the sensor readings at their desired values. At Keck the actuators are updated every $0.5 \mathrm{~s}$; for the large segmented telescopes of the future, the update rate is likely to be higher.

In this paper we consider a variety of $N$-ring telescopes as well as the nominal CELT design. An $N$-ring telescope consists of $N$ hexagonal rings of segments, with the central segment missing, for a total of $3 N^{2}+3 N$ segments. The Keck telescopes are three-ring telescopes with 36 segments each. CELT has a circularized design; that is, it starts with 20 rings, but is then circularized by exclusion of those segments whose centers lie at a distance of greater than $30.1 a$ from the optic axis, where $a$ is the hexagon side length. In addition, the central 19 segments are removed in the CELT design. The total number of CELT segments is then 1080. Figure 1 shows an 11-ring telescope as well as CELT.

In the following subsections we discuss the matrices that govern such active control systems. We discuss separately the matrices corresponding to the horizontal sensors used for the Keck telescopes and the mechanically simpler vertical sensors that have been proposed for CELT. ${ }^{11}$ As we describe, both sensor designs rely on changes in differential capacitance resulting from the relative motion of neighboring segments. Both designs are sensitive to out-of-plane displacement as well as to changes in the dihedral angle between the segments. These two designs are representative of a variety of possible sensors that can be used to make measurements of the relative segment motion.

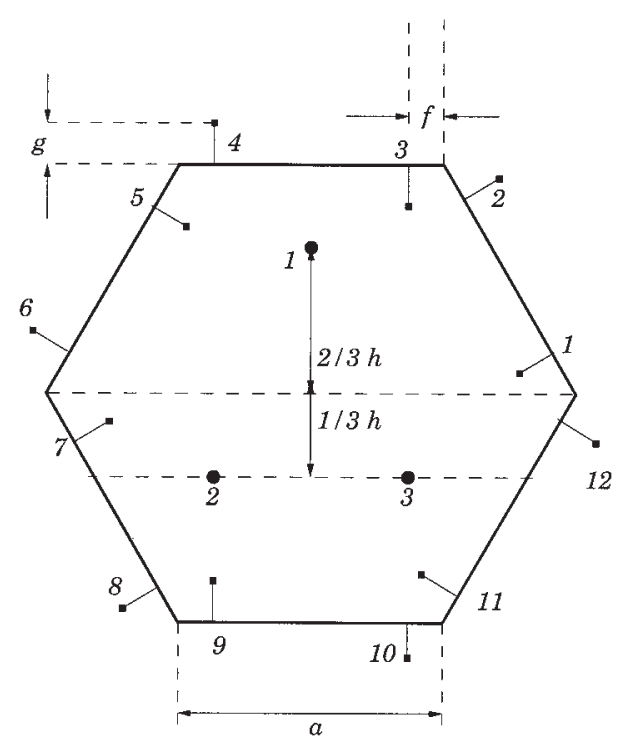

Fig. 2. Geometry of the three actuator positions and 12 sensing points on a segment.

\section{B. Construction of the Active Control Matrix for Horizontal Sensors}

The Keck sensors are horizontal, that is, the plates of the differential capacitors that make up the sensors are parallel to the segment surface. ${ }^{10}$ (The bodies of the sensors are below the lower surface of the segment.) The geometric relationships between the 12 half-sensors and the segment that they monitor are defined by Fig. 2; the placement of the three segment actuators is also indicated. The Keck parameters are given by $a=900 \mathrm{~mm}, f=173 \mathrm{~mm}, g=55 \mathrm{~mm}$, and $h=706 \mathrm{~mm}$. The sensors sense the relative edge height, that is, the height of a segment relative to its neighbor, at the points indicated by the numbered squares in Fig. 2. For the Keck geometry, and for all geometries considered in this paper, the sensing points for sensors 7 and 12 are both above the line connecting actuators 2 and 3 ; the simple sign convention below is affected if this is not the case. The values of the ratios $f / a, g / a$, and $h / a$ for Keck are close to optimal in the sense of minimal noise multiplication (see below), but they also reflect various practical considerations; that is, the precise values do not have a fundamental significance. Nevertheless, for simplicity and directness of comparison, we assume that these same ratios obtain for all cases considered in this paper, except where we explicitly take $g=0$. Other practical concerns (specifically, the interchangeability of segments) dictate that the orientation of the actuator triangle will vary from segment to segment, but for simplicity we take all actuator triangles to have the same orientation. This simplification will not change the basic properties of the associated control matrix; in particular it will have no effect at all on the error multipliers or other similar quantities derived below.

Now, if actuator 1 is pistoned by an amount $\Delta s$, the segment will rotate about a line through actuators 2 
and 3 , so that the reading of each sensor on the segment (in height units) will change by

$$
\Delta s=\frac{r \Delta z}{h},
$$

where $r$ is the perpendicular distance from the sensor to the rotation axis, and where the sign of $r$ is positive if the sensor and the actuator are on the same side of the rotation axis and negative if they are on opposite sides.

Suppose we move actuator 1 by an amount $\Delta z$. From Eq. (1) the corresponding edge height increment at sensor positions 1 through 6 will then be

$$
\begin{aligned}
& \Delta s_{1,1}=\Delta z\left(\frac{1}{3} h+f \cos 30^{\circ}-g \sin 30^{\circ}\right) / h, \\
& \Delta s_{2,1}=\Delta z\left[\frac{1}{3} h+(a-f) \cos 30^{\circ}+g \sin 30^{\circ}\right] / h, \\
& \Delta s_{3,1}=\Delta z\left(\frac{1}{3} h+a \cos 30^{\circ}-g\right) / h, \\
& \Delta s_{4,1}=\Delta z\left(\frac{1}{3} h+a \cos 30^{\circ}+g\right) / h, \\
& \Delta s_{5,1}=\Delta z\left[\frac{1}{3} h+(a-f) \cos 30^{\circ}-g \sin 30^{\circ}\right] / h, \\
& \Delta s_{6,1}=\Delta z\left(\frac{1}{3} h+f \cos 30^{\circ}+g \sin 30^{\circ}\right) / h .
\end{aligned}
$$

For sensor positions 7 through $12, \Delta s_{j, 1}$ can be obtained from $\Delta s_{j-6,1}$, but with $\sin 30^{\circ}$ and $\cos 30^{\circ}$ replaced by $-\sin 30^{\circ}$ and $-\cos 30^{\circ}$, respectively. A useful check on the signs and normalizations of the above relations is provided by the closure relations, which follow from the symmetries of the system:

$$
\begin{aligned}
\Delta s_{1,1}+\Delta s_{5,1}+\Delta s_{9,1} & =\Delta z, \\
\Delta s_{2,1}+\Delta s_{6,1}+\Delta s_{10,1} & =\Delta z, \\
\Delta s_{3,1}+\Delta s_{7,1}+\Delta s_{11,1} & =\Delta z, \\
\Delta s_{4,1}+\Delta s_{8,1}+\Delta s_{12,1} & =\Delta z .
\end{aligned}
$$

We now need to relate the edge height increments to the readings on the differential capacitors that constitute the edge sensors. The Keck edge sensors ${ }^{10}$ consist of a paddle on one segment that fits into a U-groove on its neighboring segment across the intersegment gap to form two parallel-plate capacitors, one above the other, each having a capacitance $C=\epsilon_{0} A / d$. So that all segments are similar, the U-groove is attached to the segment of interest at the odd-sensor locations in Fig. 2 and to the paddle at the even-sensor locations. As one segment moves up by an amount $\Delta s$ relative to its neighbor, the differential capacitance will change according to

$$
\frac{\Delta C_{1}-\Delta C_{2}}{C}= \pm \frac{2 \Delta s}{d}
$$

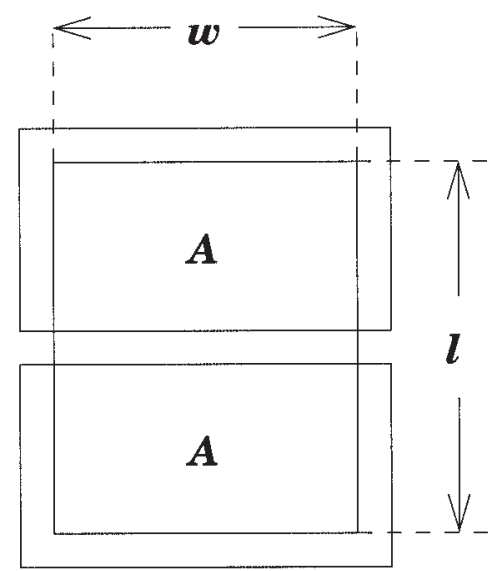

Fig. 3. Geometry of the vertical (CELT) sensors.

where the subscripts 1 and 2 denote top and bottom, respectively; the minus sign holds for those sensors with the U-groove on the segment of interest, and the plus sign holds for those sensors with the paddle on the segment of interest.

We define the sensor gain $G$ as the absolute value of the ratio $\Delta s /\left(\Delta C_{1}-\Delta C_{2}\right)$, or in terms of fixed sensor parameters,

$$
G=\frac{1}{2} \frac{d}{C}
$$

The control matrix element $A_{i j}$ is then defined to be the increase in $G\left(\Delta C_{1}-\Delta C_{2}\right)$ for the $i$ th capacitor associated with an incremental motion of $1 \mu \mathrm{m}$ of the $j$ th actuator. For actuator 1 and the sensors in Fig. 2 we have

$$
A_{j, 1}= \pm \Delta s_{j, 1} / \Delta z
$$

where the sign is positive for $j=2,4,6,7,9,11$ and negative for the remaining values of $j$. The entire control matrix can readily be filled out in this way. Each actuator affects 12 sensors (or fewer for peripheral segments because these do not have six nearest neighbors), and each sensor is affected by six actuators (in all cases). Thus each row of the control matrix has up to 12 nonzero elements, and each column has exactly six nonzero elements.

\section{Construction of the Active Control Matrix for Vertical Sensors}

The sensors proposed for CELT are vertical, that is, the capacitor plates are perpendicular to the segment surface. ${ }^{11}$ In particular, one half of the sensor consists of a single vertical sense plate bonded or plated directly onto the side of one segment, and the other half consists of two vertical drive plates on the side of its neighbor segment directly across the intersegment gap. The CELT sensor geometry is shown in Fig. 3; here $w$ is the width of the capacitor drive plates, $l$ is the height of the single plate, and $A=w l / 2$ is the area of each drive plate. We assume that the 
vertical gap between plates is small. Figure 2 still applies but with $g=0$.

In this case, unlike the horizontal sensors, there are two contributions to the differential capacitance:

$$
\frac{\Delta C_{1}-\Delta C_{2}}{C}=\frac{\Delta A_{1}-\Delta A_{2}}{A}-\frac{\Delta d_{1}-\Delta d_{2}}{d} .
$$

The first (area) term on the right-hand side of Eq. (7) is due to the shear of the segment of interest relative to its nearest neighbor across the gap; the second (rotation) term is due to the rotation of the segment relative to its neighbor, i.e., to the change in the dihedral angle. Again, to make all segments similar, the construction of the sensors should alternate as one proceeds around the segment; for the sake of definiteness, and so that the signs are similar to the above, we take the even sensors in Fig. 2 to have the double plates and the odd sensors to have the single plates.

It is easiest to consider first what happens to sensor 3 in Fig. 2 when actuator 1 is pistoned out of the page by an amount $\Delta z$. (This case is relatively simple because the associated segment edge is then parallel to the rotation axis.) Under these circumstances, Eq. (7) reduces to

$$
\frac{\Delta C_{1}-\Delta C_{2}}{C}=-\frac{4 r \Delta z}{l h}-\frac{l \Delta z}{2 h d},
$$

where all symbols are as defined in Eqs. (1) and (4). The first term on the right-hand side of Eq. (8) comes from the change in the overlap area. The second comes from the change in the effective separation of the plates.

Putting in the appropriate signs, trigonometric factors, and normalizations for all terms, and proceeding as before, we obtain the typical matrix elements:

$$
\begin{aligned}
& A_{1,1}{ }^{\prime}=-\left(\frac{1}{3} h+f \cos 30^{\circ}\right) / h-\eta \sin 30^{\circ}, \\
& A_{1,2}{ }^{\prime}=\left[\frac{1}{3} h+(a-f) \cos 30^{\circ}\right] / h-\eta \sin 30^{\circ}, \\
& A_{1,3}{ }^{\prime}=-\left(\frac{1}{3} h+a \cos 30^{\circ}\right) / h-\eta \\
& A_{1,4^{\prime}}=\left(\frac{1}{3} h+a \cos 30^{\circ}\right) / h-\eta, \\
& A_{1,5}{ }^{\prime}=-\left[\frac{1}{3} h+(a-f) \cos 30^{\circ}\right] / h-\eta \sin 30^{\circ}, \\
& A_{1,6^{\prime}}=\left(\frac{1}{3} h+f \cos 30^{\circ}\right) / h-\eta \sin 30^{\circ},
\end{aligned}
$$

where

$$
\eta=\frac{l^{2}}{8 h \delta}
$$

and the primes distinguish these results for vertical sensors from the above unprimed results for horizontal sensors. Note that $\eta$ quantifies the relative sensitivity to rotation (dihedral angle) versus shear. The matrix elements $A_{1, j}{ }^{\prime}$ with $j=7$ through 12 can be obtained from $A_{1, j-6}^{\prime}{ }^{\prime}$ with $\cos 30$ replaced by - cos $30^{\circ}$ and $\eta$ replaced by $-\eta$ (but $\sin 30^{\circ}$ is not replaced by $-\sin 30^{\circ}$ ). Note that the signs and trigonometric factors associated with the second terms on the righthand side of Eqs. (9) are determined by the orientation of the segment edges; in particular, the signs of these latter terms do not alternate as one goes around the segment. As before, from this point it is straightforward to fill out the entire control matrix. The nominal CELT segments have a circumscribed radius of $a=500 \mathrm{~mm}$ and a thickness of $50 \mathrm{~mm}$; for such segments typical sensor geometries would be $l=$ $20 \mathrm{~mm}, h=400 \mathrm{~mm}$, and $\delta=2 \mathrm{~mm}$, for a nominal value of $\eta=0.06$. In this paper we mainly consider a slightly more conservative sensor geometry defined by $\eta=0.05$.

\section{Error Propagation by the Control Matrix}

With the matrix that governs the active control system now specified, we can proceed to discuss the inversion of this system (how to obtain the desired actuator changes from the observed sensor readings) and its associated error propagation. The relation between sensors and actuators is given by

$$
\Delta s_{i}=\sum_{j} A_{i j} \Delta z_{j}
$$

where $i$ runs over all sensors and $j$ over all actuators, and where the symbol $\Delta$ refers to the difference between the actual sensor or actuator readings and their desired (absolute) readings. The desired sensor readings are defined when the alignment of the telescope is correct as determined by external optical means; actuator lengths are changed further only to maintain these desired sensor readings in the face of deformations due to gravity and temperature changes. The actuator changes that will maintain the desired sensor readings are calculated with the aid of the pseudo-inverse matrix whose elements are $B_{j i}$ :

$$
\Delta z_{j}=\sum_{i} B_{j i} \Delta s_{i}
$$

\section{Singular-Value Decomposition}

A powerful technique used to calculate the pseudoinverse matrix $\mathbf{B}$ is singular-value decomposition (SVD) of the original matrix. ${ }^{17,18}$ We briefly review this method here. In SVD the $m \times n$ matrix $\mathbf{A}$ (where $m \geq n$ ) can be written as the product of three matrices:

$$
\mathbf{A}=\mathbf{U W V}^{T},
$$

where $\mathbf{U}$ is an $m \times n$ column orthogonal matrix, $\mathbf{W}$ is an $n \times n$ diagonal matrix whose diagonal elements $w_{i}$ are positive or zero and are referred to as the singular 
values of the matrix $\mathbf{A}, \mathbf{V}$ is an $n \times n$ orthogonal matrix, and the symbol $T$ denotes transpose. The matrix $\mathbf{B}$ is then obtained as

$$
\mathbf{B}=\mathbf{V} \mathbf{W}^{-1} \mathbf{U}^{T},
$$

where the $j$ th diagonal element $1 / w_{j}$ of $\mathbf{W}^{-1}$ is replaced by 0 in the event that $w_{j}=0$. The matrix $\mathbf{V}$ defines an essentially unique orthonormal basis set of modes of the system, such that any arbitrary configuration of the system can be expressed as a unique linear combination of these modes. In particular, $V_{i j}$ gives the value of the $i$ th actuator in the $j$ th mode.

For the control matrices considered here, three of the singular values are equal to zero; the corresponding singular modes are the three actuator vectors corresponding to rigid body motion (global piston, tip, and tilt) of the primary mirror as a whole (because such motion has no effect on the sensor readings). If there are $n$ segments, there are $3 n$ actuators and $3 n-3$ modes of interest in the basis set. The normalization of the $V_{i j}$ is defined by

$$
\sum_{i} V_{i j}^{2}=1
$$

for all $j$.

\section{Error Multipliers from Singular-Value Decomposition}

If we were to put random uncorrelated noise equally into all sensors, then the actuators would respond proportionally as determined by the $A$ matrix:

$$
\delta a=\alpha \delta s,
$$

where $\delta s$ and $\delta a$ are the rms values of the sensors and actuators, and we refer to the dimensionless parameter $\alpha$ as the (overall) noise multiplier. Alternatively, we could put random noise into the sensors and determine the rms amplitude $\delta \alpha_{k}$ for each of the above $3 n-3$ modes.

By the orthogonality of the modes, we have

$$
\delta a^{2}=\sum_{k} \delta a_{k}^{2}=\sum_{k} \alpha_{k}^{2} \delta s^{2} .
$$

It is convenient to order the modes according to the size of their error multipliers, from largest to smallest. With this ordering, we define a residual error multiplier $r_{k}$, which includes the error multiplier of the $k$ th mode and all higher modes:

$$
r_{k}^{2}=\sum_{j \geq k} \alpha_{j}^{2}
$$

(Note that $r_{1}$ is then the same as the global error multiplier $\alpha$.) When the modes are ordered in this way, they are also more or less ordered in spatial frequency from lowest to highest. The reason for this correspondence is not hard to understand: Low-spatial-frequency modes have small edge discontinuities that are difficult for the sensors to detect and therefore for the active control system to control;

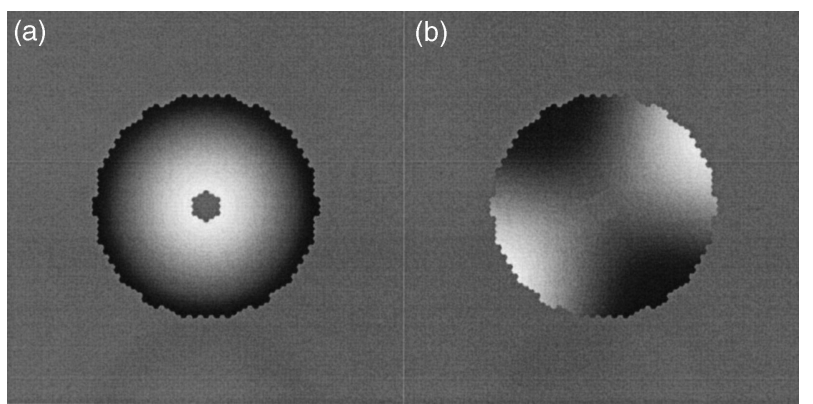

Fig. 4. Two lowest-spatial-frequency (highest error multiplier) modes for CELT, assuming vertical sensors with $\eta=0.05$ : (a) mode 1 , (b) mode 2.

high-spatial-frequency modes have large edge discontinuities that are easily detected by the edge sensors.

The error multiplier $\alpha_{j}$ associated with the $j$ th mode can be shown to be

$$
\alpha_{j}{ }^{2}=\sum_{i}\left(\frac{V_{i j}}{w_{j}}\right)^{2} .
$$

The individual error multipliers $\alpha_{j}$ and the overall error multiplier $\alpha$ are independent of the hexagon side length $a$.

This analysis is also applicable to the limiting case of vertical sensors with $\eta=0$, except that here there is an additional singular mode resulting from the fact that these sensors cannot sense a change in the intersegment dihedral angle. Thus this mode corresponds to a constant dihedral angle, with no segmentto-segment shear. Because the resulting surface resembles the error surface corresponding to an overall focus error (except that the former is faceted), it is referred to as focus mode. For sensors with nonzero $\eta$, the corresponding mode is nonsingular, but is generally the mode with the largest error multiplier.

Figure 4 shows the two CELT modes with the lowest spatial frequencies (largest error multipliers) for the case of vertical sensors with $\eta=0.05$; Figure 5 shows the two highest-spatial-frequency modes (smallest error multipliers). These results are typical of all sensor geometries (except for the extra singular mode associated with $\eta=0$ ). Inspection of the modes shows that there is a close correspondence

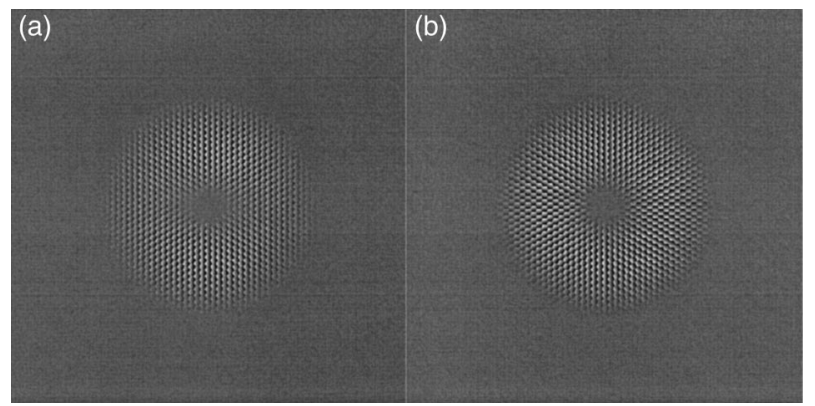

Fig. 5. Same as Fig. 4 but for the two highest-spatial-frequency (lowest error multiplier) modes: (a) mode 3236, (b) mode 3237. 


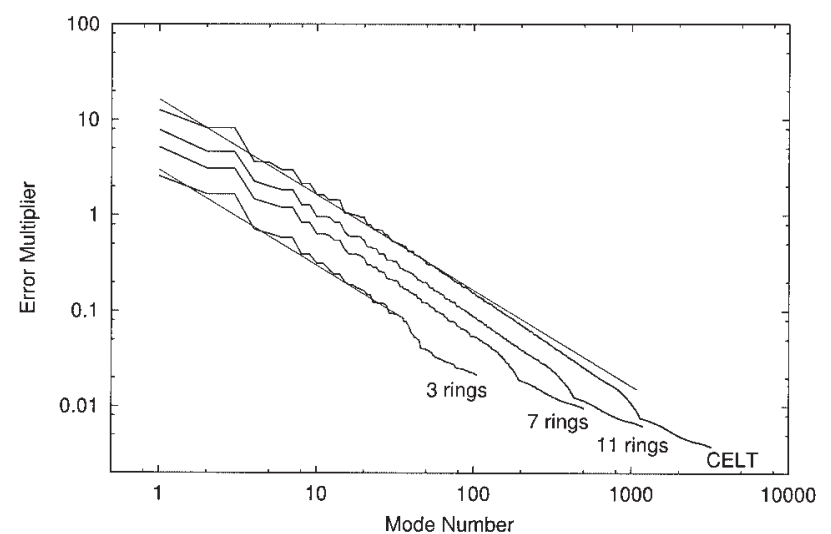

Fig. 6. Individual error multipliers for primary mirror active control systems of telescopes with 3, 7, and 11 rings and for CELT. All cases correspond to vertical sensors with $\eta=0.05$. The straight lines (plotted only for the three-ring telescope and for CELT) show that the error multipliers for the $n_{s} / 3$ lowest modes can be approximated by the function $0.5 \sqrt{n_{s}} / i$, where $n_{s}$ is the number of segments and $i$ is the mode number.

between the lowest-order modes and the Zernike polynomials.

Figure 6 shows the full range of error multipliers for 3-ring, 7-ring, and 11-ring telescopes and CELT, all with CELT-style sensors with $\eta=0.05$. Figure 7 shows a similar plot but for the residual error multiplier [see Eq. (18)]. For CELT with $\eta=0.05,84 \%$ of the overall control system noise comes from focus mode alone; $98 \%$ of the noise comes from the ten lowest modes of the system. The error multiplier depends sensitively on $\eta$ for focus mode (mode 1 ): For CELT we have $\alpha_{1}=24.5$ for $\eta=0.05$, but $\alpha_{1}=$ 12.6 for $\eta=0.10$. However, for modes 2 and higher, the error multipliers are only weakly dependent on $\eta$ (e.g., $\alpha_{2}=8.8$ for $\eta=0.05$ and $\alpha_{2}=8.2$ for $\eta=0.10$ ). The error multiplier curves scale as the square root of the total number of segments.

The sharp decline in error multiplier with increasing mode number raises the possibility of our being able to improve the mirror control by supplementing

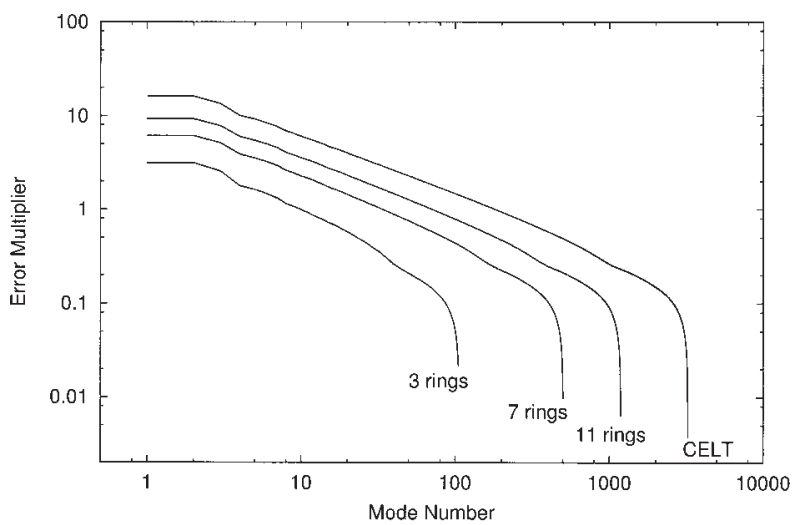

Fig. 7. Same as Fig. 6 but for residual error multipliers, defined to include the multipliers for the indicated mode and for all higherorder modes. the electromechanical sensor readings with wavefront information. We considered this question in detail elsewhere ${ }^{19,20}$ and concluded that a supplementary wave-front sensor is not likely to be needed for stabilization purposes. Here we just summarize the argument. For diffraction-limited [adaptive optics (AO)] observing, a wave-front sensor will already be present as a part of the $\mathrm{AO}$ system. If we assume that the Keck sensor noise levels of a few nanometers or less are representative of extremely large telescopes as well, the wave-front errors associated with the active control system-even allowing for the relatively large error multipliers calculated above-will be a small perturbation on the aberrations associated with atmospheric turbulence. It follows that a reasonable AO system will necessarily have the dynamic range and bandwidth to correct automatically any residual low-spatial-frequency errors left over from the active control system; supplementary alignment wave-front sensors should not be necessary. For seeing-limited observations, we will not have an $\mathrm{AO}$ wave-front sensor to correct the misalignments automatically, but the fact remains that the atmospheric aberrations will dominate by a large factor. If the image quality is tolerable in the presence of atmospheric aberrations, it is likely to be tolerable still in the presence of the additional relatively small aberrations resulting from the active control system.

The above considerations should not be taken as an argument against closed-loop focus control for large segmented telescopes. A low-bandwidth focus correction loop is motivated by considerations of control of the secondary mirror, whereas the above argument was in the context of the segmented primary. Implementation of closed-loop focus control would be a good addition to the Keck telescopes, which may still be retrofitted for this control in the future.

\section{Surface Errors from Singular-Value}

\section{Decomposition for Diffraction-Limited Observing}

For diffraction-limited observing, a useful optical figure of merit for the telescope is the rms wave-front error, which is equal to twice the rms surface error. Here we show that the rms surface error is nearly equal to the rms actuator error. As discussed above, the latter quantity is readily obtained as the overall error multiplier times the rms sensor error.

Let $z_{i j}$ represent the displacement of the $i$ th actuator $(i=1,2,3)$ on the $j$ th segment,

$$
p_{j}=\left(z_{1 j}+z_{2 j}+z_{3 j}\right) / 3
$$

represent the piston error on that segment, and $\beta_{x}$ and $\beta_{y}$ represent the angles through which the segment is rotated about the $x$ and $y$ axes. Let single brackets denote the appropriate average over the telescope; thus $\left\langle z_{1}\right\rangle$ is the average of all the $i=1$ actuators over the $n_{s}$ segments, but $\langle z\rangle$ is the average over all $3 n_{s}$ actuators. Double brackets denote the average over both the telescope and the ensemble. We take the ensemble to be defined by a Gaussian distribution of sensor (not actuator) errors, or equiv- 
alently, by the collection of SVD modes weighted by the error multipliers calculated above. Because rotating the primary mirror by $120^{\circ}$ and $240^{\circ}$ rotates the $i=1$ actuator to the $i=2$ and $i=3$ positions, various double-bracketed actuator averages cannot depend on $i$. Thus, for example, we have

$$
\left\langle\left\langle z_{1} p\right\rangle\right\rangle=\left\langle\left\langle z_{3} p\right\rangle\right\rangle=\left\langle\left\langle z_{3} p\right\rangle\right\rangle,
$$

from which it follows that

$$
\left\langle\left\langle z_{1} z_{2}\right\rangle\right\rangle=\left\langle\left\langle z_{2} z_{3}\right\rangle\right\rangle=\left\langle\left\langle z_{3} z_{1}\right\rangle\right\rangle .
$$

It follows from Eqs. (21) and (22) that the mean squared values of the various quantities will be related by

$$
\left\langle\left\langle\beta_{x}{ }^{2}\right\rangle\right\rangle=\left\langle\left\langle\beta_{y}{ }^{2}\right\rangle\right\rangle=\frac{9}{4 h^{2}}\left(\left\langle\left\langle z^{2}\right\rangle\right\rangle-\left\langle\left\langle p^{2}\right\rangle\right\rangle\right) .
$$

We let $\tilde{z}$ represent the continuous height variable over the segment surface, where $\tilde{z}=z_{i}$ at the actuator locations. Again, averaging as appropriate, we have

$$
\left\langle\left\langle\tilde{z}^{2}\right\rangle\right\rangle=\left\langle\left\langle\beta_{x}{ }^{2}\right\rangle\right\rangle\left\langle x^{2}\right\rangle+\left\langle\left\langle\beta_{y}{ }^{2}\right\rangle\right\rangle\left\langle y^{2}\right\rangle+\left\langle\left\langle p^{2}\right\rangle\right\rangle,
$$

where $x$ and $y$ are the coordinates in the nominal plane of the segment relative to an origin at the segment center. For a regular hexagon of side $a$, we have

$$
\left\langle x^{2}\right\rangle=\left\langle y^{2}\right\rangle=\frac{5}{24} a^{2},
$$

and thus

$$
\left\langle\left\langle\tilde{z}^{2}\right\rangle\right\rangle=\kappa\left\langle\left\langle z^{2}\right\rangle\right\rangle+(1-\kappa)\left\langle\left\langle p^{2}\right\rangle\right\rangle,
$$

where

$$
\kappa=\frac{15}{16} \frac{a^{2}}{h^{2}} .
$$

As noted above we take the ensemble of actuator errors to be defined by a Gaussian distribution of sensor errors; we can perform the ensemble averaging efficiently and exactly by summing over the mirror modes with the above error multipliers as weighting factors. Because the modes are strongly weighted toward low spatial frequencies, we expect that $\left\langle\left\langle z^{2}\right\rangle\right\rangle \approx\left\langle\left\langle p^{2}\right\rangle\right\rangle$ and thus $\left\langle\left\langle\tilde{z}^{2}\right\rangle\right\rangle \approx\left\langle\left\langle z^{2}\right\rangle\right\rangle$, i.e., that the actuator errors provide an excellent estimate of the overall surface error. In terms of $\mathbf{V}$ and $\mathbf{W}$ and the above formalism, we have

$$
\left\langle p^{2}\right\rangle=\frac{1}{9 n_{s}} \sum_{i j k}{ }^{\prime}\left(\frac{V_{i k} V_{j k}}{w_{k}^{2}}\right),
$$

where $V_{i k}$ refers to the value of the $i$ th actuator in the $k$ th mode, $w_{k}$ is the singular value in the $k$ th mode (unless it is equal to zero, in which case the corresponding term is omitted), $n_{s}$ is the total number of segments, and $h$ is the height of the actuator triangle as defined above. The sum is over all modes $k$ and over all actuators $i$ and $j$, with the restriction on the
Table 1. Rms Ray ${ }^{a}$ Tilt (One Dimension) per Nanometer of Sensor Noise for Various Telescopes ${ }^{b}$

\begin{tabular}{llllll}
\hline & & \multicolumn{4}{c}{ rms Tilt $(\mathrm{mas} / \mathrm{nm})$} \\
\cline { 3 - 6 } Sensor & $\eta$ & 3-ring & 7-ring & 11-ring & CELT \\
\hline Vertical & 0 & 1.60 & 1.67 & 1.74 & 1.87 \\
Vertical & 0.05 & 3.08 & 2.91 & 2.90 & 2.98 \\
Vertical & 0.10 & 1.99 & 1.96 & 1.99 & 2.09 \\
Horizontal & - & 2.29 & 2.22 & 2.24 & 2.33 \\
\hline
\end{tabular}

${ }^{a}$ The rms segment tilt is half as large.

${ }^{b}$ This assumes a segment side length of $0.500 \mathrm{~m}$.

latter (as indicated by the prime) that for a given $i$ we include only those $j$ values corresponding to an actuator on the same segment. This calculation shows that the exact ratio $\tilde{z}_{\text {rms }} / z_{\text {rms }}$ is indeed close to unity for most practical telescope geometries. However, the ratio is weakly dependent on $a$ even for constant $a / h$; for example, for the Keck geometry, the ratio is 1.028 for $0.5-\mathrm{m}$ segments and 0.971 for $0.9-\mathrm{m}$ segments. For 0.5-m segments and a telescope of seven rings (168 segments) or larger, the rms surface and actuator errors differ by less than $1 \%$ and there is little point in maintaining a distinction between these two quantities.

\section{Tip-Tilt Errors from Singular-Value Decomposition for Seeing-Limited Observing}

For seeing-limited observations, we are interested not in the rms surface error (as discussed above), but rather in the rms surface (that is, segment) tip and tilt, or the rms ray tip and tilt, which includes the factor of 2 for doubling on reflection. Here we present a prescription for obtaining the rms ray tip and tilt directly from the SVD formalism.

A straightforward extension of the preceeding analysis gives the mean-squared one-dimensional tilt in the $x$ direction as

$$
t_{x}^{2}=\frac{1}{n_{s} h^{2}} \sum_{i j k}^{\prime}\left(\frac{c_{i x} c_{j x} V_{i k} V_{j k}}{w_{k}^{2}}\right),
$$

where the $c_{i x}$ are dimensionless and defined below. The units of $t_{x}$ are radians per meter when $h$ is expressed in meters. The values of $c_{i x}$ are given by

$$
c_{1 x}=0, \quad c_{2 x}=\sqrt{3}, \quad c_{3 x}=-\sqrt{3}
$$

for the three actuators on segment 1 and similarly for actuators on all other segments. The calculation of the mean-squared $y$ tilt is the same except for the numerical values:

$$
c_{1 y}=-2, \quad c_{2 y}=1, \quad c_{3 y}=1 .
$$

A useful computational check is provided by the fact that the mean-squared tilts in $x$ and $y$ should be identical.

In Table 1 we present the one-dimensional rms ray tip and tilt per nanometer of sensor noise for various telescope and sensor geometries. Although the error multipliers discussed above were independent of 
the segment size and scaled as the square root of the total number of segments, the tip-tilt noise scales inversely as the segment size, can vary by a factor of $\sim 2$ with sensor geometry, and is virtually independent of the number of segments. Thus the typical $\mathrm{rms}$ one-dimensional image blur is $2-3$ mas per $\mathrm{nm}$ of sensor noise for a segment with $a=0.500 \mathrm{~m}$ or 1-1.5 mas $/ \mathrm{nm}$ for a segment with $a=1 \mathrm{~m}$.

\section{Alignment}

\section{A. General Considerations}

The problem of segment alignment is closely related to, but different from, the above problem of segment active control. The task of the active control system is to freeze the relative positions of the primary mirror segments in the face of perturbations due to gravity and temperature once the mirror is in the desired configuration. Conversely, it is the task of the mirror alignment system to determine the desired configuration (desired sensor readings) in the first place. The active control loop runs continuously (at $2 \mathrm{~Hz}$ for Keck, probably somewhat higher for CELT); the mirror alignment is done only infrequently-perhaps once a month, depending on the drift rate of the sensor electronics.

We have shown elsewhere ${ }^{9,21}$ that, by exploiting diffraction effects from misaligned segment edges, we can make optical edge measurements that are in some sense analogous to the electromechanical edge measurements provided by the capacitive edge sensors. One could thus approach the initial alignment problem in a way that is almost identical mathematically to the control problem analyzed above. A second approach is also possible (this is the one actually used at Keck), in which one proceeds in two steps: First, the segments are aligned in tip and tilt and then, in a separate procedure, in the piston degree of freedom. In this subsection we briefly describe these two approaches and their relative advantages.

First, suppose that there are two optical measurements per intersegment edge, made in essentially the same physical locations as the electromechanical edge sensors. The optical measurements are made exactly at the intersegment edge (that is, $g=0$ ) so that the geometry is essentially that of the vertical sensors considered above, except that in this case there is no sensitivity to rotation, only to shear. Thus the analysis summarized in Section 2 applies, with $\eta=0$. In this case there is an extra singular mode - the focus mode (in addition to the usual singular modes of global piston, tip, and tilt) - that cannot be extracted from the optical measurements, but we neglect this complication for now.

Alternatively, suppose that the segment tips and tilts are measured with a Shack-Hartmann-type scheme, ${ }^{16}$ similar to that commonly employed in AO systems. After these measurements are made and the segments are tipped and tilted appropriately, the segment pistons must still be adjusted or phased. The phasing procedure is defined mathematically as minimizing the steps between segments; this is done by means of diffraction measurements made at the centers of the intersegment edges. Relevant mathematical details are presented in Subsections 3.B and 3.C.

Now we want to determine whether, in a given circumstance, we should use the single-step alignment approach (analogous to the control approach) or the two-step approach. Let the angular uncertainty (in one dimension and as measured in arcseconds on the sky) in the Shack-Hartmann-type measurements be $\delta \theta$, the height uncertainty (as measured in nanometers at the surface) in the diffraction-type edge measurements be $\delta e$, and the ratio (from Table 1) of rms ray tilt to sensor noise (in arcseconds per nanometer) be $\mu$. The one-step versus two-step decision hinges formally on whether $\delta e$ is less than $\delta \theta / \mu$, in which case the single-step approach is favored, or greater than $\delta \theta / \mu$, in which case the two-step approach is preferred. In practice, however, the situation is more complicated because of the additional singular mode associated with the single-step approach and because of the additional optical difficulties associated with making two measurements per edge in the single-step approach, as opposed to one measurement per edge in the two-step approach. Both of these practical considerations favor the twostep method. At Keck, we have $\delta e=10 \mathrm{~nm}, \delta \theta=$ 0.030 arc sec, ${ }^{21,22}$ and $\mu=0.001$ arc $\mathrm{sec} / \mathrm{nm}$ (from Table 1, scaling to 0.9-m segments), but the two-step method is used, even though $\delta \theta / \mu=30 \mathrm{~nm}$. This decision should be reevaluated for extremely large telescopes, but we note that in general smaller segments favor the two-step approach because the corresponding values of $\mu$ are larger and the two-per-edge measurements are more difficult to make.

One could also imagine a hybrid alignment approach in which the low-spatial-frequency tip-tilt modes are measured with a Shack-Hartmann sensor and the high-spatial-frequency modes are measured by two-per-edge phase-type measurements (which would also determine the segment piston errors), but a detailed treatment of this possibility is beyond our present scope.

\section{B. Construction of the Phasing Matrix}

In this subsection we describe the control aspects of the mathematics associated with the phasing procedure, i.e., minimizing the intersegment edge steps (as measured at the edge centers). A detailed discussion of this so-called narrowband phasing algorithm can be found elsewhere. ${ }^{21}$

The control matrix for phasing is easily constructed. The $j$ th edge is determined by the values of the pistons of the adjacent segments:

$$
q_{j}=p_{i_{+}(j)}-p_{i_{-}(j)},
$$

where $i_{+}(j)$ and $i_{-}(j)$ are the indices of the segments on the positive and negative sides, respectively, of the $j$ th edge. This matrix is independent of the details of the sensor geometry. In the case of the phasing ma- 


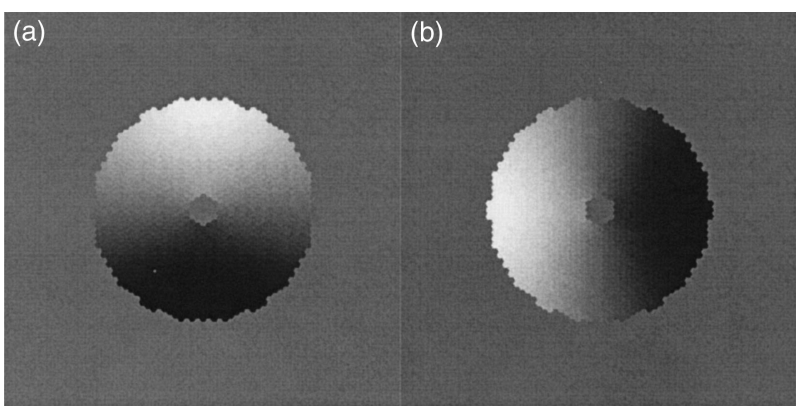

Fig. 8. Two lowest-spatial-frequency (highest error multiplier) phasing modes for CELT: (a) mode 1, (b) mode 2. Note the large tilt components.

trix, there is only one singular mode-that corresponding to global piston.

\section{Properties of the Phasing Matrix}

The phasing modes with the lowest and highest spatial frequencies are shown for the nominal CELT geometry in Figs. 8 and 9, respectively. Plots of the error multipliers (individual and residual) for all phasing modes are shown in Fig. 10 for the CELT geometry. The scaling law for the phasing matrix is different from that of the control matrix described above. In the case of the control matrix, the overall error multiplier scaled as the square root of the total number of segments or actuators; in the case of the

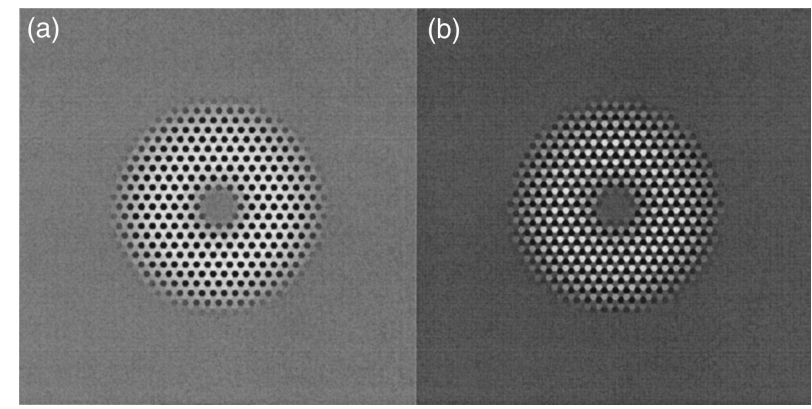

Fig. 9. Same as Fig. 8, but for the two highest-spatial-frequency (lowest error multiplier) modes: (a) mode 1078, (b) mode 1079.

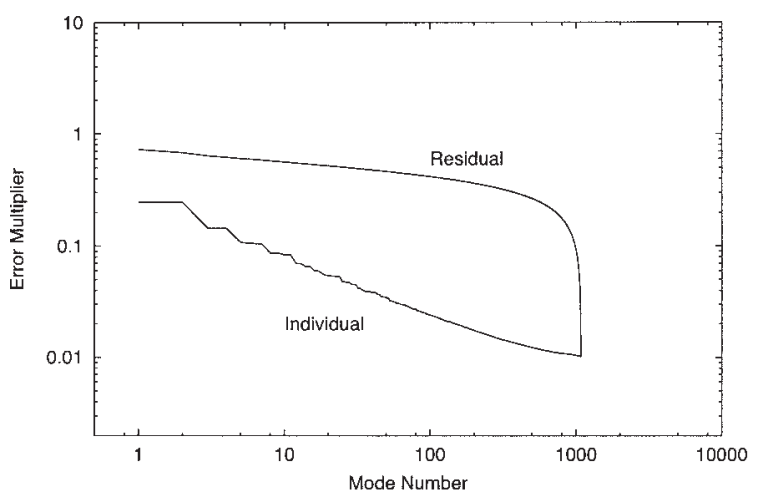

Fig. 10. Error multipliers for phasing modes of CELT: upper curve, residual multipliers (defined as in Fig. 7); lower curve, individual mode multipliers. phasing matrix, the error multiplier is almost independent of the number of segments, increasing only from 0.620 for the 36 -segment Keck geometry to 0.725 for the 1080-segment CELT geometry.

For the phasing modes (as was the case for control modes), the error multipliers fall off rapidly with increasing spatial frequency, so that we expect the spatial-frequency content of the residual piston errors after piston alignment to be significantly lower than that of uncorrelated piston errors. For one consequence of this, note from Fig. 8 that modes 1 and 2 resemble global coma, for which the best-fit plane has a nonzero slope. Because these modes are the largest contributors to the overall error multiplier, this means that the best-fit plane to the overall residual phase errors will have a larger slope than that for random phase errors. Thus a fraction of the residual wave-front error associated with phasing is indistinguishable from global tilt. For the Keck geometry, subtracting off the best-fit plane will reduce the rms piston error by approximately $20 \%$; for the CELT geometry, the corresponding reduction is approximately $12 \%$. In quoting phasing errors at Keck, we have generally subtracted off this tip-tilt component. For similar reasons, the deformable mirror in an AO system may help to mitigate phasing errors, depending on how the wave-front sensor deals with the wave-front discontinuities.

Experience at Keck has shown that the segment tip and tilts need to be adjusted considerably more often than the segment pistons. ${ }^{22}$ The question then arises: What set of actuator commands will correct the segment tip and tilts while having the smallest deleterious effects on the segment pistons (as the segments will not always be phased immediately following tip-tilt adjustment)? This question is an important one because the least-stable, lowest-order modes are in fact dominated by segment pistons. Conceptually, it is simplest to break up the solution into two steps. (1) Determine the actuator commands that will zero out the segment tip and tilts, subject to the constraint that the mean actuator length change for each segment is zero. (This can always be done exactly.) Also determine the change in all the intersegment edge heights implicit in these actuator commands. The actuator commands are then sent. (2) Determine the (pure) piston commands that will remove, or at least minimize, the edge height changes predicted in the previous step. This can be done with the phasing matrix determined above. Sending these latter commands will produce the optimal configuration of the mirror for these given circumstances.

\section{Summary and Conclusions}

We have given a general prescription for the construction of the mirror control matrices and also for the phasing matrices for essentially arbitrary highly segmented mirrors. We have described the modes and error propagation for both types of matrix. In the mirror control matrix, the overall error multiplier scales as the square root of the number of segments, 
which may present a problem for telescopes with several thousand segments. The only singular modes associated with the control matrices are the three global rigid body degrees of freedom, plus one additional singular mode in the limiting case of very short vertical sensors $(\eta=0)$, which are not sensitive to changes in the intersegment dihedral angle. In the phasing matrix, the overall error multiplier is virtually independent of the number of segments, which suggests that straightforward extensions of current phasing techniques should be adequate even for the largest of the extremely large telescopes. Although further analysis is needed, it is possible, if not likely, that segment tip-tilt alignment and phasing of extremely large telescopes will best be carried out in two separate procedures, as is currently done for the Keck telescopes. We have argued elsewhere that supplemental wave-front control, in terms of an auxiliary wave-front sensor, should not be needed for the primary mirror, although a low-order systemessentially an enhanced guider-is probably necessary to keep the secondary mirror properly aligned.

We thank Mitchell Troy and Lothar Noethe for many useful discussions.

\section{References}

1. J. E. Nelson, "Progress on the California Extremely Large Telescope (CELT)," in Future Giant Telescopes, J. R. P. Angel and R. Gilmozzi, eds., Proc. SPIE 4840, 47-59 (2002).

2. T. Anderson, A. L. Ardeberg, J. Beckers, A. Goncharov, M. Owner-Petersen, H. Riewaldt, R. Snel, and D. Walker, "The Euro-50 extremely large telescope," in Future Giant Telescopes, J. R. P. Angel and R. Gilmozzi, eds., Proc. SPIE 4840, 214-225 (2002).

3. J. R. P. Angel, J. H. Burge, J. L. Codona, W. B. Davison, and B. Martin, "20- and 30-m telescope designs with potential for subsequent incorporation into a track-mounted pair (20/20 or 30/30)," in Future Giant Telescopes, J. R. P. Angel and R. Gilmozzi, eds., Proc. SPIE 4840, 183-193 (2002).

4. D. Burgarella, K. Dohlen, M. Ferrari, F. Zamkotsian, F. Hammer, F. Sayede, and R. Rigaut, "Large petal telescope for the next-generation Canada-France-Hawaii Telescope," in Future Giant Telescopes, J. R. P. Angel and R. Gilmozzi, eds., Proc. SPIE 4840, 93-103 (2002).

5. P. Dierickx, J. L. Beckers, E. Brunetto, R. Conan, E. Fedrigo, R. Gilmozzi, N. N. Hubin, F. Koch, M. Lelouarn, E. Marchetti, G. J. Monnet, L. Noethe, M. Quattri, M. S. Sarazin, J. Spyromilio, and N. Yaitskova, "The eye of the beholder: designing the OWL," in Future Giant Telescopes, J. R. P. Angel and R. Gilmozzi, eds., Proc. SPIE 4840, 151-170 (2002).

6. S. C. Roberts, C. L. Morbey, D. R. Crabtree, R. Carlberg, D. Crampton, T. J. Davidge, J. T. Fitzsimmons, M. H. Gedig, D. J. Halliday, J. E. Hesse, R. G. Herriot, J. B. Oke, J. S. Pazder, K. Szeto, and J.-P. Veran, "Canadian very large optical telescope technology studies," in Future Giant Telescopes, J. R. P. Angel and R. Gilmozzi, eds., Proc. SPIE 4840, 104-115 (2002).

7. S. E. Strom, L. M. Stepp, and B. Gregory, "Giant Segmented Mirror Telescope: a point design based on science drivers," in
Future Giant Telescopes, J. R. P. Angel and R. Gilmozzi, eds., Proc. SPIE 4840, 116-128 (2002).

8. R. Cohen, T. Mast, and J. Nelson, "Performance of the W. M. Keck Telescope active mirror control system," in Advanced Technology Optical Telescopes V, L. M. Stepp, ed., Proc. SPIE 2199, 105-116 (1994).

9. G. A. Chanan, M. Troy, F. G. Dekens, S. Michaels, J. Nelson, T. Mast, and D. Kirkman, "Phasing the mirror segments of the Keck telescopes: the broadband phasing algorithm," Appl. Opt. 37, 140 (1998).

10. J. E. Nelson, T. S. Mast, and S. M. Faber, "The design of the Keck Observatory and Telescope," Keck Observatory Rep. No. 90 (W. M. Keck Observatory, Kamuela, Hawaii, 1985).

11. J. Nelson, ed., "The California Extremely Large Telescope," CELT Rep. No. 34 (University of California and California Institute of Technology, Santa Cruz, Calif., 2002).

12. T. Mast and J. Nelson, "Segmented mirror control system hardware for CELT," in Optical Design, Materials, Fabrication, and Maintenance, P. Dierickx, ed., Proc. SPIE 4003, 226 240 (2000).

13. J. Nelson, "CELT segment positioning actuatorsrequirements," CELT Tech. Note No. 5 (University of California, Santa Cruz, Santa Cruz, Calif. 2001).

14. T. G. Barnes, M. T. Adams, J. A. Booth, M. E. Cornell, N. I. Gaffney, J. R. Fowler, G. J. Hill, G. M. Hill, C. E. Nance, F. Piche, L. W. Ramsey, R. L. Ricklets, W. J. Spiesman, and P. T. Worthington, "Commissioning experience with the 9.2-m Hobby-Eberly Telescope," in Telescope Structures, Enclosures, Controls, Assembly/Integration/Validation, and Commissioning, T. A. Sebring and T. Andersen, eds., Proc. SPIE 4004, $14-25$ (2000).

15. P. Alvarez, J. M. Rodriguez Espinoza, and F. R. Kabana, "GTC project: present and future," in Telescope Structures, Enclosures, Controls, Assembly/Integration/Validation, and Commissioning, Proc. SPIE 4004, 26-35 (2000).

16. G. A. Chanan, "Design of the Keck Observatory alignment camera," in Precision Instrument Design, T. C. Bristow and A. E. Hathaway, eds., Proc. SPIE 1036, 59-70 (1988).

17. W. Press, B. Flannery, S. Teukolsky, and W. Vetterling, $\mathrm{Nu}$ merical Recipes: the Art of Scientific Computing (Cambridge U. Press, New York, 1989), pp. 484-487.

18. E. Anderson, Z. Bai, C. Bischof, J. Demmel, J. Dongarra, J. D. Croz, A. Greenbaum, S. Hammarling, A. McKenney, S. Ostrouchov, and D. Sorensen, LAPACK User's Guide, 2nd ed. (Society for Industrial and Applied Mathematics, Philadelphia, Pa., 1995)

19. D. G. MacMartin and G. A. Chanan, "Control of the California Extremely Large Telescope primary mirror," in Future Giant Telescopes, J. R. P. Angel and R. Gilmozzi, eds., Proc. SPIE 4840, 69-80 (2002).

20. D. G. MacMartin and G. Chanan, "Measurement accuracy in control of segmented-mirror telescopes," Appl. Opt. (to be published).

21. G. A. Chanan, C. Ohara, and M. Troy, "Phasing the mirror segments of the Keck Telescopes: the narrowband phasing algorithm," Appl. Opt. 39, 4706-4714 (2000).

22. M. Troy, G. Chanan, E. Sirko, and E. Leffert, "Residual misalignments of the Keck telescope primary mirror segments: classification of modes and implications for adaptive optics," in Advanced Technology Optical/IR Telescopes VI, L. M. Stepp, ed., Proc. SPIE 3352, 307-317 (1998). 